\title{
Research on the Computer Graphics Rendering Technology based on GPU and Parallel Computing System
}

\author{
Honglin Huang \\ Guangzhou International Economics College, Guangdong 510540, China
}

Keywords: Computer Graphics, Rendering, GPU, Parallel Computing, Systematic Design.

\begin{abstract}
In this paper, we conduct research on the computer graphics rendering technology based on GPU and parallel computing system. Traditional geometry based on the polygon rendering technique, due to difficulty in modeling and rendering time is too long, has been more and more cannot adapt to the large-scale complex scene accurately modeling and real-time display. On the other hand, with the improvement of image acquisition equipment precision and falling prices is easy to get high precision of image information, based on image rendering technology gradually become one of the main means of scene rendering. Under this basis circumstance we propose the new perspective of the methodology that will optimize the traditional ways and provide systematic modification.
\end{abstract}

\section{Introduction}

Computer graphics image processing technology is to use the basic composition of the graphics image processing software and primary computer hardware equipment. Generally speaking, the higher the performance of the computer hardware equipment, more can deal with some high quality graphics, the graphics image processing software to display the terminal will be computer closely together. For commonly used computer graphics image processing software, because of it itself has that modified, storage and design, and other functions which can quickly integrate together data from the image, thus greatly lighten the burden of running central processing unit. Computer image processing technology in the practical application range is very wide, its implementation is mainly depends on the function of the computer aided. In actual application of the computer has high processing speed and accuracy of the real, save, powerful wait for the characteristic, therefore, using the computer image processing technology also has very many advantage that is illustrated in the figure one.

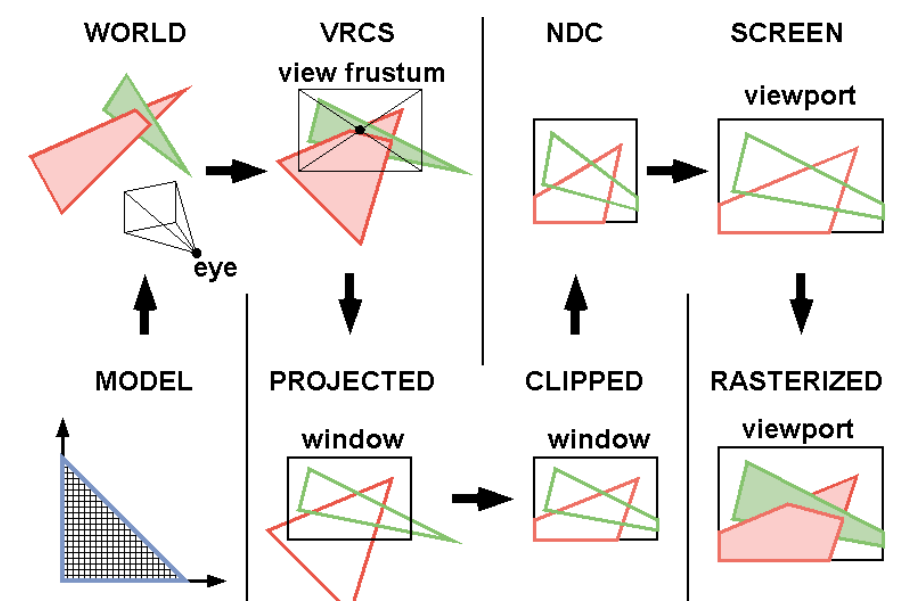

Figure 1. The General Transformation of the Computer Graphics

Computer image not realistic rendering technology as a new basic technology, high attention by all aspects, the current can achieve pencil drawing, the oil painting, watercolor painting and other artistic effect of the image, the performance of the computer image non-realistic rendering technique for imitating the painter's creation and process images using filtering technology, the need to deal with the user interaction, while the latter is by the different smoothing and sharpening template to filter the image, thus achieve image of stylized processing [1-2]. 
The characteristics of the digital media has developed rapidly has huge development prospects. In many industries, digital media has been widely used, and the sustainable development. Our artistic brushwork rendering algorithm is not the purpose of the study, and but one can use all kinds of artistic rendering algorithm of the general three-dimensional image rendering framework it can automatically convert 3D scene to all kinds of artistic style of stereo images and can ensure the consistency of the binocular images and the novelty of our paper can be listed as follows. (1) Used for image texture mapping all the information should include binocular images, we presents the binocular vision image acquisition method. (2) Shows an independent of the specific artistic rendering algorithm of the stereo image generation framework. Using the method of texture mapping is directly on the surface model generated art brush. In the later sections, we will discuss the issues in detail.

\section{The Proposed Algorithm}

Computer Graphics Rendering. Computer image of realistic rendering technique has until now has always been the focus of the international conference, and with the efforts of numerous experts and scholars, has achieved outstanding results. Using computer image not real rendering technology is not only able to draw up the art style of the 2D floor plan that also can extend the non-real images of 3D.

For 3D virtual scene generate stereo images is the most direct method, that respectively, using the horizontal two cameras around eyes images. If used in projection before the model artistic processing, make the model surface with an artistic touch, you can directly get artistic stereo images. Realistic image of the model which we call "reference image" as the reference image contains objects and light color, shade and depth of the information, such as the basis of the information is add artistic brush. Because the model need to be done from the perspective of two different projection imaging, so the reference images and brushwork texture must contain two viewpoints can be seen. In addition, in the process of generating brushwork texture, the texture coordinates must be generated at the same time model that is really easy to perform the subsequent texture mapping.

Simulation program operation process that is actually a frame by frame to complete the scene rendering and display and if you want to complete the scene image processing operations, must be rendered in the scene is good but has not yet been completed before display of scene read that image processing and writing frame cache the three steps back as the figure two.

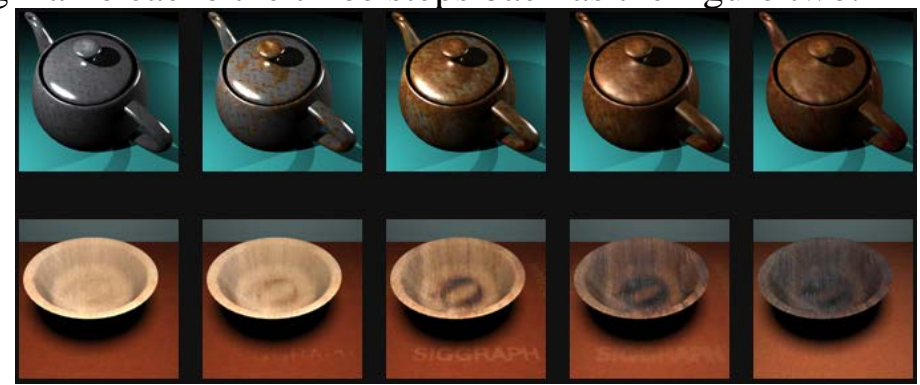

Figure 2. The Demonstration of the Computer Graphics Rendering Technology

Parallel Computing System. Massively parallel computing is the first step in the data assigned to each computer, distributed computing each processor alone a child tasks, not to be shared, because the data parallel computing adjacent area data needs to be in the process of mutual exchange, through the messaging communication between the processor.

Development in field of structure analysis of parallel algorithm is of great practical significance. At present, the parallel algorithm is applied in the field of structural analysis there are two main direction of solving linear equation of the parallel and parallel solution based on substructure. Direct solving method is mainly for linear algebraic equation on its parallelism and substructure method of parallel solving is seeking substructure on structure divided between parallelisms in order to obtain parallel task assignment for many of the large-scale scientific and engineering computing problem, a high performance computer can not only meet the demand as they need to be geographically 
distributed, heterogeneous, a variety of the computing resources through high-speed Internet connection, common computational problems. However, the complexity of the application model is often more than the current workstations real-time processing ability the traditional distributed parallel computing system huge expenditure of energy in the algorithm design [3-7].

The popularization of computer network application also broke through the traditional can only use a computer to solve the limitation can through the cooperation to solve several computers as this was born the parallel computing and its algorithm as the so-called parallel computing refers to a single executable multiple operating calculation, the research of parallel computing, now has developed into an independent field of study, many with serial calculation algorithms to solve the questions also have corresponding parallel computing algorithm and with the rapid improvement in parallel processing hardware performance which could be reflected from the following figure 3.

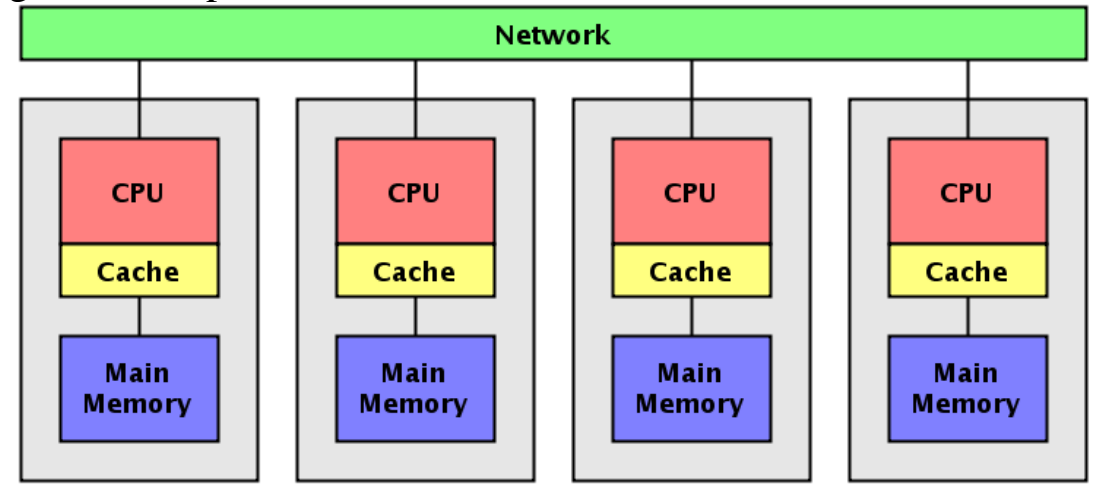

Figure 3. The Demonstration of the Parallel Computing System

GPU and Features. Often compared to the GPU in computing speed CPU can get the acceleration of one or several orders of magnitude. Due to the physical structure of the CPU and GPU is different, so the CPU and basic GPU for floating-point operation ability have very big difference. The GPU is designed for data intensive, computational intensive and highly parallel computing. GPU parallel image points on rows and columns to prefix addition to complete, so improve the GPU integral image of a prefix is the key to build speed addition.

Absorbing traditional primary software architecture description language program design in the semantic characteristics of strict and precise, and for the integrity of the software architecture and the abstract, define and determine the suitable for software architecture expression and description about the abstract elements, which can be precise, unambiguous to describe the software architecture, better support for general software architecture refinement, validation, evolution and analysis. Target level processing is operated on produced by a series of characteristics of target. Because of the complexity and symbolic target information is usually carried out using relevant knowledge reasoning as for the description of the image, understanding, explanation and general recognition.

Animation Design Framework. Traditional animation production adopted by the way is going to the dynamic part of the decomposed into multiple action, then every move designed to draw out one by one, only the slight difference between the adjacent pictures while again as will these actions play continuously formed animation effects and because of the color in the traditional animation design based on the principle of reflective color, so in the absence of the light color will become dark, compared with the Flash animation design in general concentration, purity and brightness of color performance has a great advantage.

Also in terms of color changes, the traditional two-dimensional animation color not only need a lot of time to form a preview, at the same time under the condition of the color appears a certain deviation is not easy to modify, and in Flash animation design, only need to use the software can quickly color and form a preview, at the same time also can quickly change the color. Current animation that is widely used in the basic development environment is a Java and Flash. The method of using Java environment animation are diverse, its main principle is through the calculation language to make a series of movement of the frame, to realize the image motion. In the mobile digital media animation design, most of the technical personnel selection using Canvas class tool software 
animation effects. Canvas can be according to certain speed, running a set of pictures to create the animation effect.

Computer Graphics Skills Optimization. Refers to the computer image processing technology, using computer technology to deal with image analysis, image contains information are obtained for research institute. That is to say, this technology is that the main points of the first, will have to study image transformation, into computers able to identify the "digital matrix", because only in this way, can store image to a computer; Second, the stored in the computer image processing, among them, all kinds of calculation method is the most common methods for processing. Finally, after the processing of related technologies, it is concluded that the research of image information. The reduction of image and enhanced in many cases, the need of image processing, such as eliminating noise so as to realize the reduction and image enhancement, in order to get higher quality of the image as follows.

- Image enhancement. In order to make more high quality digital images, the need for image enhancement operation, make the image more meticulous, so that the computer analysis of the recognition. To this, the main point of the enhancement is to realize high resolution image.

- Image restoration. Due to the influence of some factors, such as environment, equipment and other factors, cause image distortion or fuzzy. So, original image is bad for image information acquisition, and the need for image processing, improve the quality of the image.

- Gray level histogram processing. At present, the processing method has been widely used, also has the good image enhancement effect, such as fog image processing as is widely used in the method. This processing method is to use the gray level histogram image processing of the contrast is not strong, and then realize the small local processing clearly.

\section{Summary}

In this paper, we conduct research on the computer graphics rendering technology based on GPU and parallel computing system. IBR for scene rendering puts forward a new point of view, and is a kind of high efficient rendering technique, and a large number of the data source information, higher input equipment requirements, and not well deal with the scene in various lighting effects is the main drawback of this technology. Add/remove some information in the scene is one of the technical difficulties, and as the lack of the support of the basic hardware rendering technique makes the IBR technology rendering speed seems not so satisfactory. In the later research, we will combine more related machine learning and pattern recognition to enhance the current approach.

\section{References}

[1] Cavalcanti, M. G. P., S. S. Rocha, and M. W. Vannier. "Craniofacial measurements based on 3D-CT volume rendering: implications for clinical applications." Dentomaxillofacial Radiology.

[2] Rousselle, Fabrice, Claude Knaus, and Matthias Zwicker. "Adaptive rendering with non-local means filtering." ACM Transactions on Graphics (TOG) 31.6 (2012): 195.

[3] Ma, Bingpeng, Yu Su, and Frédéric Jurie. "Bicov: a novel image representation for person re-identification and face verification." British Machive Vision Conference. 2012.

[4] Srinivas, Umamahesh, et al. "Simultaneous sparsity model for histopathological image representation and classification." Medical Imaging, IEEE Transactions on 33.5 (2014): 1163-1179.

[5] Wang, Haoxiang, and Jingbin Wang. "An effective image representation method using kernel classification." Tools with Artificial Intelligence (ICTAI), 2014 IEEE 26th International Conference on. IEEE, 2014.

[6] Hong, Xiaopeng, et al. "Capturing Correlations of Local Features for Image Representation." Neurocomputing (2015). 
[7] Zheng, Yunping, and Mudar Sarem. "A novel binary image representation algorithm by using NAM and coordinate encoding procedure and its application to area calculation." Frontiers of Computer Science 8.5 (2014): 763-772. 